\title{
Nosocomial outbreak of staphyloccocal scalded skin syndrome in neonates in England, December 2012 to March 2013
}

K Paranthaman (karthik.paranthaman@phe.gov.uk) ${ }^{1,2}$, A Bentley ${ }^{3}$, L M Milne ${ }^{4}$, A Kearns 5 , S Loader ${ }^{3}$, A Thomas ${ }^{3}$, F Thompson ${ }^{3}$, M Logan ${ }^{6}$, S Newitt ${ }^{7}$, R Puleston 7

1. Public Health England, East Midlands Public Health England Centre, Leicester, United Kingdom

2. Public Health England, Kent, Surrey and Sussex Public Health England Centre, Ashford, United Kingdom (current affiliation)

3. Northampton General Hospital NHS Trust, Northampton, United Kingdom

4. Public Health England, South Midlands and Hertfordshire Public Health England Centre, Letchworth Garden City, United Kingdom

5. Public Health England, Staphylococcus Reference Service, London, United Kingdom

6. Public Health England, Midlands, Birmingham, United Kingdom

7. Public Health England, Field Epidemiology Service, Nottingham, United Kingdom

Citation style for this article:

Paranthaman K, Bentley A, Milne LM, Kearns A, Loader S, Thomas A, Thompson F, Logan M, Newitt S, Puleston R. Nosocomial outbreak of staphyloccocal scalded skin syndrome in neonates in England, December 2012 to March 2013. Euro Surveill. 2014;19(33):pii=20880. Available online: http://www.eurosurveillance.org/ ViewArticle.aspx?Articleld $=20880$

Article submitted on 13 January 2014 / published on 21 August 2014

Staphylococcal scalded skin syndrome (SSSS) is a blistering skin condition caused by exfoliative toxin-producing strains of Staphylococcus aureus. Outbreaks of SSSS in maternity settings are rarely reported. We describe an outbreak of SSSS that occurred among neonates born at a maternity unit in England during December 2012 to March 2013. Detailed epidemiological and microbiological investigations were undertaken. Eight neonates were found to be infected with the outbreak strain of $S$. aureus, of spa type t346, representing a single pulsotype. All eight isolates contained genes encoding exfoliative toxin A (eta) and six of them contained genes encoding toxin B (etb). Nasal swabs taken during targeted staff screening yielded a staphylococcal carriage rate of $21 \%$ (17/80), but none contained the outbreak strain. Mass screening involving multi-site swabbing and pooled, enrichment culture identified a healthcare worker (HCW) with the outbreak strain. This HCW was known to have a chronic skin condition and their initial nasal screen was negative. The outbreak ended when they were excluded from work. This outbreak highlights the need for implementing robust swabbing and culture methods when conventional techniques are unsuccessful in identifying staff carrier(s). This study adds to the growing body of evidence on the role of HCWs in nosocomial transmission of $S$. aureus.

\section{Introduction}

Staphylococcal scalded skin syndrome (SSSS) predominantly but not exclusively affects neonates and children under the age of five years. It results from infection with strains of Staphylococcus aureus containing genes encoding exfoliative toxin A (eta) and B (etb). Around $5 \%$ of all $S$. aureus produce exfoliative toxins. These toxins act in the zona granulosa of the epidermis, leading to a spectrum of illness ranging from mild localised blistering to extensive generalised lesions [1]. The prognosis in most cases is typically good if prompt antibiotic therapy is given [2].

The incidence rate of SSSS in England is unknown as no national clinical or laboratory surveillance system is in place. A review of the Staphylococcal Reference Unit database of Public Health England showed that around 50-100 cases of SSSS are confirmed in England each year, which equates to $0.94-1.88 \mathrm{cases} / \mathrm{million} / \mathrm{year}$ (unpublished data). Incidence of SSSS probably varies among countries in Europe, with estimates ranging from 0.56 cases/million/year in France to 2.53 cases/ million/year in the Czech Republic [3,4].

Outbreaks of SSSS have been infrequently reported in maternity and neonatal units [5-7]. Transmission in these outbreaks was interrupted by the identification and management of symptomatic infection or asymptomatic colonisation of healthcare staff shedding the outbreak strain and through reinforcing standard infection control measures $[7,8]$.

Four neonates born within one week of each other at a maternity unit in England developed SSSS in 2013. The first affected neonate in the outbreak was born in December 2012. All four neonates were readmitted at the age of 8-9 days and were found to be infected with tetracycline-resistant $S$. aureus with genes encoding exfoliative toxin A and B. For the purpose of the investigation, the putative exposure period for acquisition of the outbreak strain in the neonate was the time from birth till discharge home. The outbreak period spanned 
from the birth of the first affected neonate to the date of symptom onset in the last affected neonate (in March 2013). Day o of the outbreak period refers to the day before the birth of Case 1. An Outbreak Control Team (OCT), chaired by the Director of Infection Prevention and Control, was convened, with representation from hospital and public health staff. The hypothesis for the cause of the outbreak was an exposure or exposures occurring in the maternity unit from birth until discharge home. We describe the investigation and control measures that led to the resolution of the outbreak.

\section{Methods}

\section{Setting}

The outbreak occurred in an acute hospital with approximately 100 maternity beds. The maternity unit performs about 4,400 deliveries per year and has a dedicated labour ward with two operating theatres. Two dedicated wards for antepartum and postpartum care, with a total of about 40 beds, are located at a short distance from the labour ward but within the main hospital site. The maternity unit has about 300 doctors, nursing, midwifery and support staff.

\section{Clinical investigations}

During the outbreak period, all neonates presenting with clinical scalded skin syndrome were identified by senior paediatric clinicians and were promptly notified to key members of the OCT. Umbilical swabs, along with swabbing of infective lesions when present, were performed in all suspected cases. Digital photography of skin lesions was also undertaken.

\section{Epidemiological investigations}

As part of case finding, the hospital microbiology records were interrogated to check whether tetracycline-resistant $S$. aureus had been isolated in the laboratory in the previous six months. Using a standardised questionnaire tool, data were collected from the medical case records of the mother and neonate on key variables such as demographics, ward locations, chronological course, antenatal, perinatal and postnatal interventions for the mother and neonate, and key staff involved in providing clinical care. Social network analysis software (i2 Analysts Notebook 8 - i2 Ltd) was used to identify staff with a high level of contact with the affected neonates, with data extracted from in-depth case note review.

\section{Environmental investigations}

Throughout the outbreak period, an enhanced programme of environmental audit was undertaken to assess standards of cleanliness and disinfection. Limited environmental sampling was undertaken on day 68 from a variety of sites $(n=28)$ such as baby cots, trolleys, incubators, resuscitaire equipment and internal and external air vents. A formal review of infection control practices and the maternity wards was conducted by a team of specialists in infection control.
Neonatal screening investigations

To determine if asymptomatic colonisation with the outbreak strain was occurring, prospective umbilical swab screening of all neonates born at the maternity unit was undertaken at the time of first discharge to home between days 29 and 35 (phase 1). This exercise was repeated between days 62 and 71 (phase 2).

\section{Staff screening investigations}

Surveillance of the hands of all maternity staff was conducted and those with known or active dermatitis (identified by occupational health records, staff members volunteering information at the time of assessment and infection control staff identifying skin problems at assessment) were referred to occupational health for clinical assessment and swabbing as appropriate.

Staff known to have been involved in the care of the affected neonates were identified and screened for $S$. aureus nasal carriage in a targeted screening exercise undertaken between days 22 and 78. Subsequently, mass screening of all maternity staff was instigated on day 81 , with a multiple site (nose, throat and hair-line) swabbing approach. All staff voluntarily complied with the request for being screened.

\section{Microbiological investigations}

Charcoal swabs were used to sample lesions from suspected cases and for screening of staff and neonates. Swabs from cases and staff in the targeted screening exercise were cultured by direct inoculation onto colistin-nalidixic acid (CNA) agar [9]. In the mass screening exercise, the pooled swabs were initially pre-enriched overnight in a brain-heart infusion before inoculation onto CNA agar. Identification and confirmation of $S$. aureus was by colony appearance, followed by latex agglutination and Gram staining [10]. Antibiotic sensitivities were conducted at the local hospital laboratory (where outbreak occurred) using the automated VITEK 2 system (bioMérieux, Inc. Hazelwood, United States).

All isolates of $S$. aureus were referred to the Staphylococcal Reference Service (Public Health England, Colindale) for detailed characterisation including the following: detection of 14 toxin genes (exfoliative toxins A, B and D; detection of enterotoxins $A-E$ and $G-J$; detection of toxic shock syndrome toxin and Panton-Valentine leucocidin, Staphylococcus protein A gene (spa) typing and pulsed-field gel electrophoresis [11].

\section{Infection control measures}

During the course of the investigations, the following control measures were implemented: (i) daily hand hygiene audits in maternity and paediatric wards and tailored interventions to achieve $100 \%$ compliance with hospital standards; (ii) enhanced environmental cleaning of all clinical areas including theatres; (iii) named individual responsibility for cleaning and equipment disinfection in theatres and other clinical areas; (iv) decommissioning of the birthing pool on the labour 
Clinical and microbiological characteristics of neonates with staphylococcal scalded skin syndrome born at a maternity unit in England, December 2012-March 2013

\begin{tabular}{|l|c|c|c|c|c|l|l|l|} 
Case & $\begin{array}{c}\text { Duration of stay in } \\
\text { hospital post-birth }\end{array}$ & $\begin{array}{c}\text { Age when } \\
\text { admitted with } \\
\text { SSSS }\end{array}$ & \multicolumn{1}{|c|}{ Birth } & Swab site & $\begin{array}{l}\text { Tetracycline } \\
\text { sensitivity }\end{array}$ & $\begin{array}{l}\text { spa } \\
\text { type }\end{array}$ & $\begin{array}{l}\text { PFGE } \\
\text { pattern }\end{array}$ & $\begin{array}{l}\text { Exfoliative } \\
\text { toxin genes }\end{array}$ \\
\hline 1 & 17 hours & 9 days & Vaginal delivery & Umbilicus, face, groin & Resistant & t346 & Pattern A & eta,etb \\
\hline 2 & 2 days, 4hrs & 9 days & Elective C-section & Umbilicus, groin & Resistant & t346 & Pattern A & eta, etb \\
\hline 3 & 16 hours & 8 days & Vaginal delivery & Umbilicus, face, groin & Resistant & t346 & Pattern A & eta,etb \\
\hline 4 & 1 day, 23 hours & 9 days & Elective C-section & Groin & Resistant & t346 & Pattern A & eta, etb \\
\hline 5 & 4 days, 12 hours & Not admitted & Emergency C-section & Umbilicus & Resistant & t346 & Pattern A & eta, etb \\
\hline 6 & 4 days, o hours & 10 days & Emergency C-section & Umbilicus, skin & Sensitive & t346 & Pattern A & eta \\
\hline 7 & 2 days, 16 hours & 9 days & Emergency C-section & Umbilicus, skin, nose & Sensitive & t346 & Pattern A & eta, etb \\
\hline 8 & 10 hours & 6 days & Vaginal delivery & Neck, groin & Sensitive & t346 & Pattern A & eta \\
\hline
\end{tabular}

PFGE: pulsed-field gel electrophoresis; SSSS: staphylococcal scalded skin syndrome.

ward; (v) removal of any communal products, such as baby bathing liquid and cotton wool; (vi) joint environmental and infection control inspections by the infection control team, with instruction or intervention provided where areas of poor practice were identified; (vii) immediate isolation and prompt treatment of neonates admitted with suspected SSSS in paediatric wards; (viii) prompt decolonisation of staff with octenidine $(0.3 \%)$ wash and mupirocin $(2 \%)$ nasal ointment when $S$. aureus was isolated in screening, without awaiting strain characterisation; (ix) letters sent by email to community health professionals (via service managers), informing them about the outbreak and advising them on the need to refer suspected cases for urgent hospital assessment; and ( $x$ ) informing all maternity and paediatric staff of the outbreak and reminding them of the importance of infection control measures.

\section{Case definition}

All clinically suspected cases were included in the outbreak from day 20, when the first meeting of the OCT was held following declaration of an outbreak. The case definition was reviewed and refined periodically by the OCT over the course of the outbreak to ensure that it remained a robust tool to delineate outbreak and non-outbreak cases based on clinical, epidemiological and microbiological information.

At the conclusion of the outbreak, the final case definition was agreed on: an SSSS case was a neonate born after day o of the outbreak at the maternity unit of the hospital, presenting with clinical scalded skin syndrome and infected with $S$. aureus genes eta and etb or eta, of spa type $\mathrm{t} 346$ belonging to multilocus sequence type clonal complex 15 (MLST CC-15) with a specific pulsotype (designated pattern A).

\section{Results}

Eight neonates met the outbreak case definition. Their key characteristics are presented in the Table. All cases were born at full-term and developed skin lesions predominantly in the perineum or periumbilical area or both within 10 days of birth. Seven were admitted to the hospital for administration of intravenous antibiotics. Case 5 was identified in phase 1 of the neonatal screening exercise and had developed a mild clinical illness by the time microbiological characterisation confirmed infection with the outbreak strain. This case was managed with oral antibiotics in the community. All neonates recovered uneventfully.

On the basis of the exposure period of the neonates in the maternity unit, the outbreak occurred in three distinct clusters - four cases in the first cluster between days 1 and 11, three cases in a second cluster between days 23 and 31 and a final 'cluster' of one case on day 88 (Figure).

Two mothers reported skin infections (breast lesion and caesarean wound infection) but their wound swabs were negative for Staphylococcus. No other illness among the mothers was reported.

A review of records of the microbiology laboratory did not reveal any tetracycline-resistant $S$. aureus isolates among neonates in the six months before the outbreak. Epidemiological investigations did not identify any common antenatal, intranatal or postnatal exposures. Possible exposures in the community (outside of the maternity unit) were considered and discarded due to lack of epidemiological evidence.

\section{Neonatal screening investigations}

In phase 1 (neonatal screening at discharge), 19/47 screened neonates were found to be colonised with $S$. aureus, of whom one (Case 5) was identified as having the outbreak strain. In phase 2 (the repeat screening), 30/67 neonates were found to be colonised with S. aureus, but none had the outbreak strain.

\section{Staff screening investigations}

Surveillance of staff members' hands identified three healthcare workers ( $\mathrm{HCWs)} \mathrm{with} \mathrm{dermatitis,} \mathrm{who} \mathrm{were}$ 
Timeline of staphylococcal scalded skin syndrome outbreak, England, December 2012-March 2013

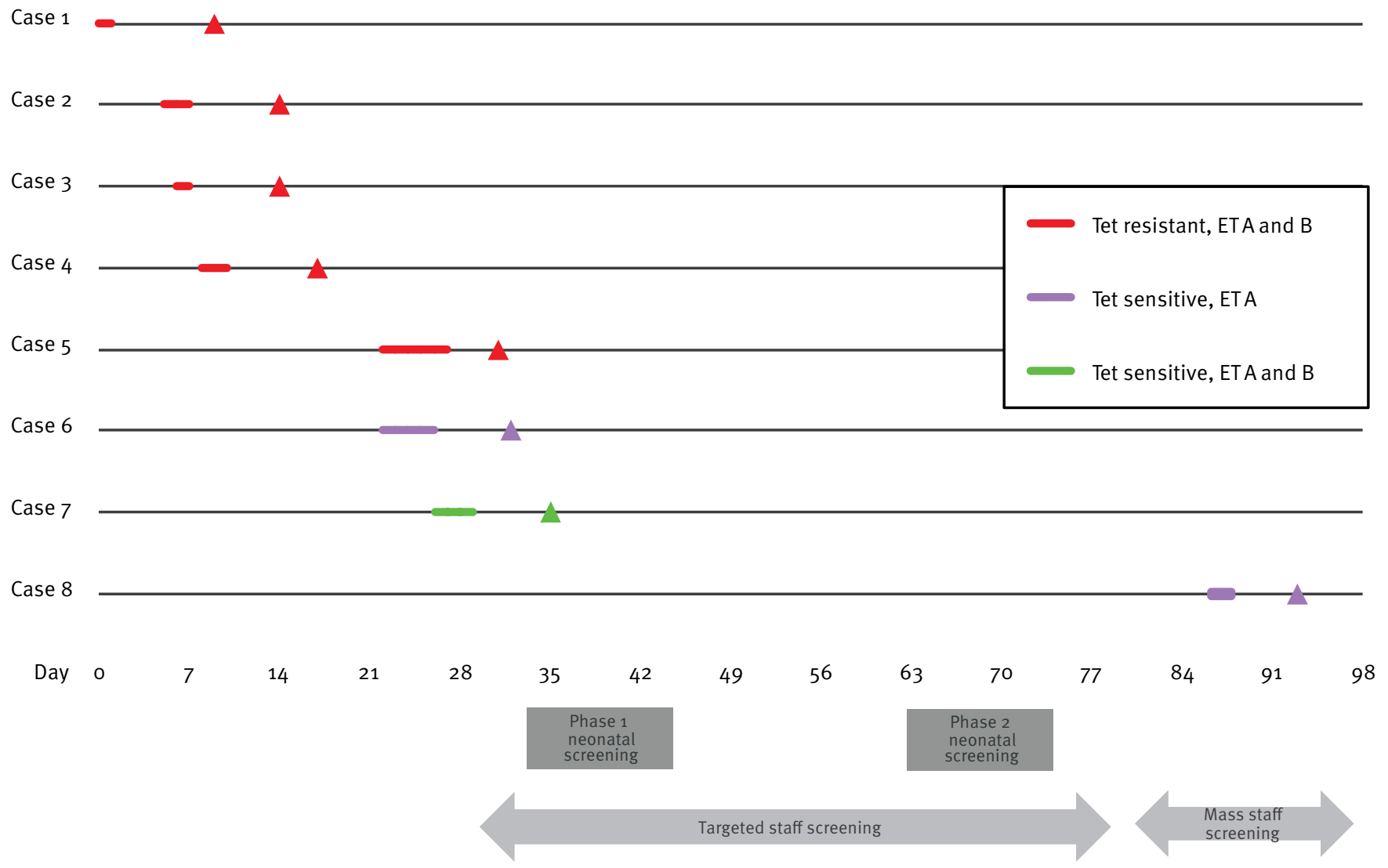

ET: exfoliative toxin; Tet: tetracycline.

The coloured bars indicate the time from birth to discharge for each case. The triangles represent the date of admission of the neonate to hospital with symptoms of staphylococcal scalded skin syndrome. Case 5 was not admitted.

referred to occupational health. Among these, one HCW had active skin lesions but a swab taken from the HCW's hand was negative for Staphylococcus. The other two HCWs were not swabbed as they did not have active dermatitis at the time of the assessment.

Targeted screening of 80 staff members using nasal swabs revealed tetracycline-sensitive staphylococcal carriage in 17 staff members. These 17 isolates were unfortunately discarded, as tetracycline resistance was initially considered as a marker for the outbreak strain. Once it became apparent that tetracycline resistance was not a reliable marker (as later cases were tetracycline sensitive), rescreening of the 17 staff members revealed the continued presence of $S$. aureus in 14 of them, but none had the outbreak strain.

A total of 20 staff members with the highest level of interaction with the cases were identified from a network analysis of the first seven cases. Since all staff members, except one, in this list had already been screened in the targeted screening exercise, the OCT decided to progress to mass screening of all maternity staff.

Mass screening of 217 maternity staff revealed the presence of Staphylococcus in 46 (21\%). Among these, a HCW (HCW A) was found on day 89 to carry the outbreak strain. Network analysis confirmed that among all staff, HCW A had had the second-highest level of interaction with the affected neonates. Of interest, HCW A was one of three HCWs with known dermatitis reviewed by the occupational health department within a week of recognition of the outbreak but did not undergo microbiological screening as they had no active skin lesions. HCW A also underwent nasal swab screening in the initial targeted screening exercise on day 22 , but $S$. aureus was not isolated.

On finding that HCW A carried the outbreak strain, they were promptly excluded from the clinical environment. Screening of the remaining maternity staff was discontinued following identification of HCW A with the outbreak strain. 
Decolonisation of HCW A was attempted: the first round failed. Following that, screening of their family contacts confirmed the presence of outbreak strain in household members. A second attempt at decolonisation of HCW A, along with their family members, also failed. Following this, HCW A was redeployed to a nonclinical role in the maternity unit around day 180.

Routine clinical and laboratory surveillance led to the recognition on day 347 of a neonate, born on day 338 at the maternity unit, with clinical SSSS and infected with the outbreak strain (tetracycline-sensitive $S$. aureus, encoding eta and etb gene, spa type t346 and pulsotype pattern A). Despite lack of evidence that HCW A had had any contact with the affected neonate, HCW A was further redeployed outside the maternity unit following this case. No further cases with the outbreak strain have been reported to date (August 2014).

\section{Environmental investigations}

All environmental swabs were negative for S. aureus. Improvements were made in cleaning, disinfection and infection control procedures as a result of the joint environmental and infection control inspections and the external review of infection control practices.

\section{Non-outbreak case}

One neonate was confirmed with SSSS associated with a different strain: eta positive, spa type t2649 (belonging to MLST-CC88) and a different pulsotype (designated pattern B). Of note, a staff member (HCW B) involved in the care of this neonate, with a previous negative nasal screen, was found to carry the same strain of $S$. aureus on rescreening. HCW B was promptly excluded and successfully decolonised. No further neonates or staff members were identified with this non-outbreak strain to date (August 2014).

\section{Discussion}

To the best of our knowledge, this is the first reported outbreak of SSSS in neonates in a maternity setting in England. Investigations were complicated by the phenotypic and genotypic variation observed in the outbreak strain, specifically the loss of tetracycline resistance and/or the etb gene in the isolates from the last three cases, most likely reflecting plasmid loss over time [12]. Furthermore, the wide variation in the antenatal, perinatal and postnatal exposures among affected neonates and mothers precluded targeting investigations and interventions at a specific ward, staff group or procedure.

Network analysis software was used in this outbreak to evaluate complex interactions between patients and HCWs. Network analysis is highly dependent on the quality of the data sources (i.e. recording of staffpatient interactions in case records) and the technical skills to analyse and interpret the data are sparse. While the software assists in determining the most likely path of interaction, interpretation becomes difficult when there are non-sequential interactions.
Despite these limitations, this technology may have application for the investigation of outbreaks where the links between cases and staff exposures are difficult to ascertain with conventional epidemiological techniques.

Individuals with skin lesions are more prone to be colonised with and likely to shed Staphylococcus [13-15]. Asymptomatically colonised HCWs with chronic skin conditions have been previously identified as sources of outbreaks of staphylococcal infection in nurseries and hospitals [6,7,16-19].

HCW A reported a history of chronic dermatitis and was previously known to occupational health. At the time of the outbreak, HCW A was not known to have any difficulties in practising routine hand hygiene measures required for providing clinical care. In retrospect, a key lesson learnt from this outbreak was that HCWs with known or active skin conditions should be screened microbiologically as well as clinically, as part of nosocomial staphylococcal outbreak investigations among neonates. HCW A underwent nasal swabbing in the targeted screening exercise on day 22 but cultures for S. aureus were negative. Possible explanations for a negative culture include intermittent colonisation or shedding, low-level carriage or inadequate sampling methodology [20-23]. Multisite swabbing with pooled, enrichment culture was ultimately successful in isolating the outbreak strain from HCW A, thus concluding the outbreak investigations. On the basis of microbiological and epidemiological evidence, it is likely that HCW A had a key role in transmission of the pathogen in this outbreak. However, the source of the outbreak strain in HCW A remains obscure as HCW A had been employed in the same clinical role in the hospital for many years and had not been implicated in previous outbreaks.

Screening of all neonates was undertaken in two distinct short periods, to understand if asymptomatic infection with the outbreak strain was occurring. Among 114 neonates screened at the time of discharge, 49 (43\%) were already colonised with S. aureus and one neonate was identified with the outbreak strain. These figures are consistent with previous studies demonstrating a staphylococcal carriage rate of $30-90 \%$ in neonates in hospitals [24-26]. Despite identifying only a single case in this instance, screening of neonates at discharge can provide valuable insight into assessing transmission rates and provide a degree of assurance on the effectiveness of infection control measures.

As all neonates had lesions centred in the umbilicus and perineum, umbilical cord care procedures were reviewed. It was noted that no specific interventions were undertaken in cord care, in line with standard national practice [27]. Antiseptics have been recommended in the past, but current consensus does not support the routine use of antiseptic agents, particularly in high-income countries [28]. 
The limitations of this study must be noted. First, the actual number of cases could have been higher. Despite our letters advising community health professionals to refer suspected cases to hospital, it is possible that some mild cases could have been managed in the community and not reported to the OCT. Second, umbilical swab screening of the neonates in the two separate phases was incomplete as all neonates discharged in the two screening phases could not be screened due to operational reasons. Nevertheless, the random nature of bias in this screening exercise is unlikely to substantially alter the rates of staphylococcal carriage found in this cohort. Third, the role of the inanimate environment in propagating this outbreak cannot entirely be excluded as environmental swabbing was limited to areas thought to be high-risk by the infection control team and was conducted late in the outbreak, following substantial improvements in cleaning and disinfection procedures.

Despite the multiple challenges encountered, continued investigations ultimately led to the identification and hence exclusion of the HCW carrying the outbreak strain thus successfully resolving the outbreak. This outbreak highlights the value of implementing robust swabbing and culture methods when conventional techniques are unsuccessful in identifying staff carrier(s). Furthermore, this outbreak adds to the growing body of evidence on the role of HCWs in the nosocomial transmission of $S$. aureus, prompting the need to reassess occupational health screening policies to monitor the health of healthcare staff, especially those with chronic dermatitis.

\section{Acknowledgements}

The authors would like to thank the support provided by the following: the Outbreak Control Team members, staff working in maternity, microbiology, infection control, Paediatrics and Occupational Health Departments in Northampton Hospital, Dr Emma Hendry, Health Protection staff in East Midlands and South Midlands \& Hertfordshire PHE centres.

\section{Conflict of interest}

None declared.

\section{Authors' contributions}

$\mathrm{KP}, \mathrm{AB}, \mathrm{LM}, \mathrm{SL}, \mathrm{AT}$ and FT were key members of the Outbreak Control Team and were involved in agreeing the strategic and operational approach to investigations and management of the outbreak. AK provided expert advice on outbreak strategy and oversaw detailed molecular characterisation of the isolates. ML provided significant input in to outbreak management strategy and led the review of infection control practices at the maternity unit. RP and SN led on the epidemiological investigations. KP wrote the first draft of the manuscript and all authors contributed to the final version.

\section{References}

1. Dimond RL, Wolff HH, Braun-Falco O. The staphylococcal scalded skin syndrome. An experimental histochemical and electron microscopic study. Br J Dermatol. 1977;96(5):483-92. http://dx.doi.org/10.1111/j.1365-2133.1977.tb07150.x

2. Gemmell CG. Staphylococcal scalded skin syndrome. J Med Microbiol. 1995;43(5):318-27. http://dx.doi.org/10.1099/00222615-43-5-318

3. Lamand V, Dauwalder O, Tristan A, Casalegno JS, Meugnier H, Bes M, et al. Epidemiological data of staphylococcal scalded skin syndrome in France from 1997 to 2007 and microbiological characteristics of Staphylococcus aureus associated strains. Clin Microbiol Infect. 2012;18(12): E514-21.

4. Lipový B, Brychta P, Chaloupková Z, Suchánek I. Staphylococcal scalded skin syndrome in the Czech Republic: an epidemiological study. Burns. 2012;38(2):296-300. http://dx.doi.org/10.1016/j.burns.2011.08.005

5. O'Connell NH, Mannix M, Philip RK, MacDonagh-White C, Slevin B, Monahan R, et al. Infant Staphylococcal scalded skin syndrome, Ireland, 2007--preliminary outbreak report. Euro Surveill. 2007;12(24):pii=3220.

6. El Helali N, Carbonne A, Naas T, Kerneis S, Fresco O, Giovangrandi Y, et al. Nosocomial outbreak of staphylococcal scalded skin syndrome in neonates: epidemiological investigation and control. J Hosp Infect. 2005;61(2):130-8 http://dx.doi.org/10.1016/j.jhin.2005.02.013

7. Occelli P, Blanie M, Sanchez R, Vigier D, Dauwalder O, Darwiche A, et al. Outbreak of staphylococcal bullous impetigo in a maternity ward linked to an asymptomatic healthcare worker. J Hosp Infect. 2007;67(3):264-70. http://dx.doi.org/10.1016/j.jhin.2007.08.023

8. Piechowicz L, Garbacz K, Budzynska A, Dabrowska-Szponar M. Outbreak of bullous impetigo caused by Staphylococcus aureus strains of phage type $3 \mathrm{C} / 71$ in a maternity ward linked to nasal carriage of a healthcare worker. Eur J Dermatol. 2012;22(2):252-5.

9. Baron EJ, Peterson LR, Finegold SM. Bailey \& Scott's diagnostic microbiology. 9th ed. St. Louis, MO: Mosby-Year Book, Inc.; 1994.

10. Public Health England (PHE). Identification of Staphylococcus species, Micrococcus species and Rothia species. UK standards for microbiology investigations. London: PHE; March 2014. Available from: http://www.hpa.org.uk/webc/ hpawebfile/hpaweb_c/1317131055573

11. Holmes A, Ganner M, McGuane S, Pitt TL, Cookson BD, Kearns AM. Staphylococcus aureus isolates carrying PantonValentine leucocidin genes in England and Wales: frequency, characterization, and association with clinical disease. J Clin Microbiol. 2005;43(5):2384-90. http://dx.doi.org/10.1128/JCM.43.5.2384-2390.2005

12. Kato F, Kadomoto N, Iwamoto Y, Bunai K, Komatsuzawa $H$, Sugai M. Regulatory mechanism for exfoliative toxin production in Staphylococcus aureus. Infect Immun. 2011;79(4):1660-70. http://dx.doi.org/10.1128/IAl.00872-10

13. Aly R, Maibach HI, Shinefield HR. Microbial flora of atopic dermatitis. Arch Dermatol. 1977:113(6):780-2. http://dx.doi.org/10.1001/archderm.1977.01640060076008 http://dx.doi.org/10.1001/archderm.113.6.780

14. Nilsson E, Henning C, Hiörleifsson ML. Density of the microflora in hand eczema before and after topical treatment with a potent corticosteroid. J Am Acad Dermatol. 1986;15(2 Pt 1):192-7.

http://dx.doi.org/10.1016/S0190-9622(86)70155-2

15. Leyden JJ, Marples RR, Kligman AM. Staphylococcus aureus in the lesions of atopic dermatitis. Br J Dermatol. 1974;90(5):52530.

http://dx.doi.org/10.1111/j.1365-2133.1974.tbo6447.x

16. Hoeger PH, Elsner P. Staphylococcal scalded skin syndrome: transmission of exfoliatin-producing Staphylococcus aureus by an asymptomatic carrier. Pediatr Infect Dis J. 1988;7(5):340-2. http://dx.doi.org/10.1097/00006454-198805000-00010

17. Dave J, Reith S, Nash JQ, Marples RR, Dulake C. A double outbreak of exfoliative toxin-producing strains of Staphylococcus aureus in a maternity unit. Epidemiol Infect. 1994;112(1):103-14.

http://dx.doi.org/10.1017/S0950268800057460

18. Haill C, Fletcher S, Archer R, Jones G, Jayarajah M, Frame J, et al. Prolonged outbreak of meticillin-resistant Staphylococcus aureus in a cardiac surgery unit linked to a single colonized healthcare worker. J Hosp Infect. 2013;83(3):219-25. http://dx.doi.org/10.1016/j.jhin.2012.11.019

19. Vonberg RP, Stamm-Balderjahn S, Hansen S, Zuschneid I, Ruden $\mathrm{H}$, Behnke M, et al. How often do asymptomatic healthcare workers cause methicillin-resistant Staphylococcus 
aureus outbreaks? A systematic evaluation. Infect Control Hosp Epidemiol. 2006;27(10):1123-7.

http://dx.doi.org/10.1086/507922

20. Wertheim HF, Melles DC, Vos MC, van Leeuwen W, van

Belkum A, Verbrugh HA, et al. The role of nasal carriage

in Staphylococcus aureus infections. Lancet Infect Dis.

2005;5(12):751-62.

http://dx.doi.org/10.1016/S1473-3099(05)70295-4

21. Nouwen J, Boelens H, van Belkum A, Verbrugh H. Human factor in Staphylococcus aureus nasal carriage. Infect Immun. 2004;72(11):6685-8.

http://dx.doi.org/10.1128/IAl.72.11.6685-6688.2004

22. Nouwen JL, Ott A, Kluytmans-Vandenbergh MF, Boelens HA, Hofman A, van Belkum A, et al. Predicting the Staphylococcus aureus nasal carrier state: derivation and validation of a “culture rule". Clin Infect Dis. 2004;39(6):806-11. http://dx.doi.org/10.1086/423376

23. Kalmeijer MD, van Nieuwland-Bollen E, Bogaers-Hofman D, de Baere GA. Nasal carriage of Staphylococcus aureus is a major risk factor for surgical-site infections in orthopedic surgery. Infect Control Hosp Epidemiol. 2000;21(5):319-23. http://dx.doi.org/10.1086/501763

24. Seeberg S, Brinkhoff B. Epidemiology and control of staphylococcal pyoderma among newborn infants: evaluation of a method for routine cord care with 4 per cent chlorhexidinedetergent solution. J Hosp Infect. 1984;5(2):121-36 http://dx.doi.org/10.1016/0195-6701(84)90116-6

25. Dancer SJ, Simmons NA, Poston SM, Noble WC. Outbreak of staphylococcal scalded skin syndrome among neonates. J Infect. 1988;16(1):87-103 http://dx.doi.org/10.1016/S0163-4453(88)96249-4

26. Seeberg S, Brinkhoff B, John E, Kjellmer I. Prevention and control of neonatal pyoderma with chlorhexidine. Acta Paediatr Scand. 1984;73(4):498-504.

http://dx.doi.org/10.1111/j.1651-2227.1984.tbo9961.x

27. National Institute for Health and Clinical Excellence (NICE).

Routine postnatal care of women and their babies. NICE; 2006. London: NICE clinical guideline 37. Available from: http://www. nice.org.uk/nicemedia/pdf/CG37NICEguideline.pdf

28. Imdad A, Bautista RM, Senen KA, Uy ME, Mantaring JB zrd, Bhutta ZA. Umbilical cord antiseptics for preventing sepsis and death among newborns. Cochrane Database Syst Rev. 2013;5:CDoo8635. 